\title{
Cruveilhier's Unrecognized Case (c1831) of Dyke-Davidoff-Masson Syndrome
}

\author{
Douglas J. Lanska ${ }^{a, b}, c$ \\ aProfessor, Institute of Social Science, I.M. Sechenov First Moscow State Medical University, Ministry of Health

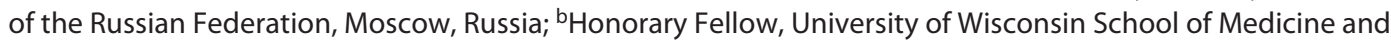 \\ Public Health, Madison, WI, USA; 'C Chair, History and Archives Committee of the American Academy of Neurology, \\ Minneapolis, MN, USA
}

\author{
Keywords \\ History of neurology · Cerebral hemiatrophy . \\ Dyke-Davidoff-Masson syndrome · Crossed \\ cerebral-cerebellar atrophy
}

\begin{abstract}
In his serially published atlas of pathology, Anatomie Pathologique du Corps Humain (1829-1842), French anatomist and pathologist Jean Cruveilhier (1791-1874) provided an early clinical-pathologic description of Dyke-DavidoffMasson syndrome. Cruveilhier's case was initially published around 1830, more than a century before the clinical and radiologic report of Dyke and colleagues in 1933 based on a series of patients studied with pneumoencephalography. Although Dyke and colleagues were apparently unaware of Cruveilhier's prior description, Cruveilhier's case manifested all of the key osseous and neuropathological features of Dyke-Davidoff-Masson syndrome as later elaborated by Dyke and colleagues: (1) cerebral hemiatrophy with ex vacuo dilation of the lateral ventricle, (2) ipsilateral thickening of the diploe of the skull, and (3) ipsilateral hyper-pneumatization of the frontal sinuses. In addition, Cruveilhier noted crossed cerebral-cerebellar atrophy in his case and correctly
\end{abstract}

inferred a "crossed effect" between the involved cerebral hemisphere and the contralateral cerebellum. Cruveilhier's pathological case from 1830 clearly anticipated both the cases reported more than a century later by Dyke and colleagues based on pneumoencephalography and the more recent case reports recognized with computed tomography or magnetic resonance imaging.

(c) 2021 S. Karger AG, Basel

\section{Introduction}

French anatomist and pathologist Jean Cruveilhier (1791-1874) (Fig. 1), the "prince of...physician-pathologists" ([1], p. 207) studied medicine at the University of Montpellier, graduated in 1816, then became chair of anatomy at the University of Paris in 1825, before ultimately becoming the first chair of pathological anatomy (i.e., pathology) in Paris in 1836. Cruveilhier possessed an extensive knowledge of morbid anatomy and published a series of multivolume works on the subject, the most important of which was the two-volume atlas of pathology Anatomie Pathologique du Corps Humain (1829-1842; Pathological Anatomy of the Human Body) [2]. This
Correspondence to:

Douglas J. Lanska, douglas.lanska@gmail.com 


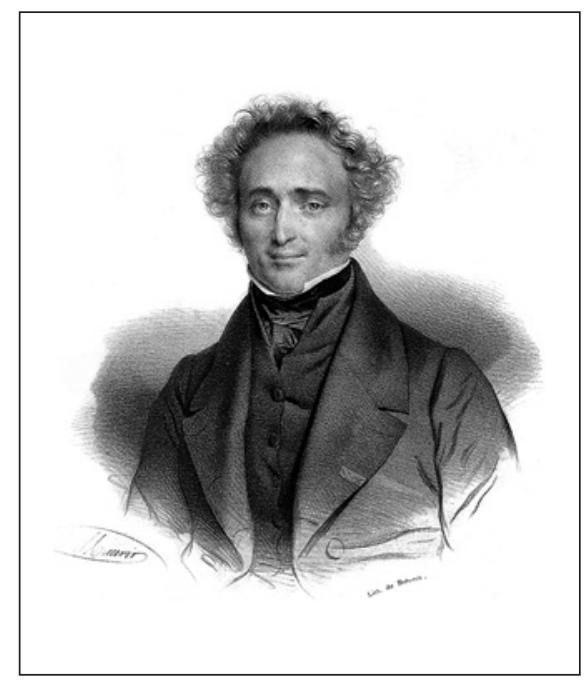

Fig. 1. French anatomist and pathologist Jean Cruveilhier (17911874 ) in 1837. Courtesy of Wellcome Images Iconographic Collection. Creative Commons Attribution 4.0 International (https:// creativecommons.org/licenses/by/4.0/deed.en) license. The image was adjusted for contrast and repaired where damaged using Apple Aperture v3.6.

work includes 233 exquisitely detailed lithograph plates ( 2 folding and 167 hand-colored) by anatomical illustrator Antoine Chazal (1793-1854), concerning a diverse collection of pathological conditions. It was originally issued in 40 fascicles (livraisons - i.e., issues or parts) to 400 subscribers over a period of 13 years, with the parts ultimately bound together into 2 volumes, the first completed in 1835 and the second in 1842. This approach was apparently chosen to allow preparation of illustrations based on the cases Cruveilhier had on hand, so the organization of the completed atlas appears somewhat haphazard. The included cases have a clinical summary with history and examination results, autopsy findings, a variable number of associated figures with corresponding legends, and Cruveilhier's insightful "reflections" on the significance of the cases.

In Anatomie Pathologique du Corps Humain, Cruveilhier gave the first clinical-pathologic description of multiple sclerosis (though without a clear history of relapsing and remitting symptoms), provided an early account of progressive muscular atrophy (sometimes called Cruveilhier's atrophy or Cruveilhier's disease), and included clinical-pathological reports of various other neurological conditions, including meningitis, intracerebral hemorrhage, vestibular schwannoma (acoustic neuroma), and intracranial and spinal meningiomas [1-3]. In addition, among Curveilhier's neurologic cases was an unrec-

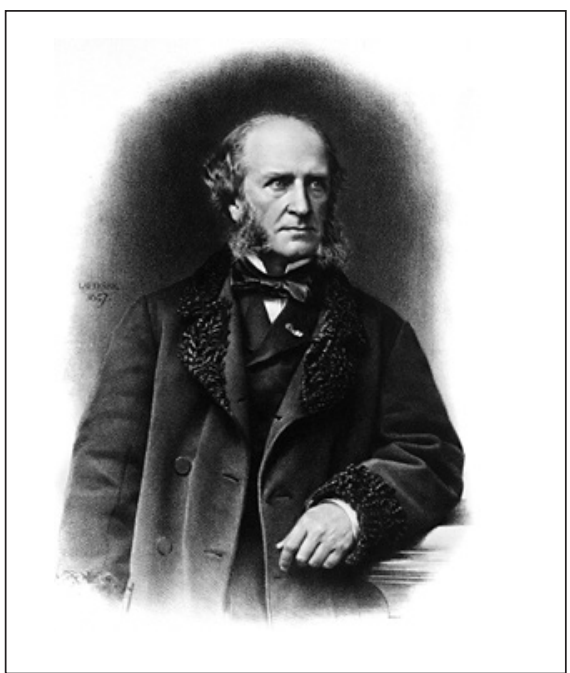

Fig. 2. French physician and surgeon Noël-Francois-Odon Guéneau de Mussy (1813-1885) at the Hotel-Dieu in Paris (1867). Lithograph by LaFosse from a photograph by Pierson. Courtesy of the US National Library of Medicine. Public domain.

ognized case of Dyke-Davidoff-Masson syndrome, more than a century before the report by Dyke and colleagues that brought this condition to clinical attention [4].

\section{Cruveilhier's Case of Dyke-Davidoff-Masson}

Syndrome (Livraison VIII, Plate V)

In the eighth fascicle of Anatomie Pathologique $d u$ Corps Humain [2], Cruveilhier presented a case which would now be recognized as a case of Dyke-DavidoffMasson syndrome. Cruveilhier had received the pathological material and the clinical observations on this case from Dr. Noël-Francois-Odon Guéneau de Mussy (18131885) (Fig. 2), a French physician and surgeon at the Hôtel-Dieu in Paris.

\begin{abstract}
Alexandre-Sylvain Augé, aged 42, unmarried, was brought to the Hotel-Dieu on February 13, 1830, with all the symptoms of a heart disease carried to the highest degree. He was moreover affected, from his most tender childhood, by an incomplete right hemiplegia of which he did not recall the beginning. The paralyzed limbs are wasted and atrophied.... Moreover, in the manner in which he answered the questions, which were made to him, and ... expressed his desires, his intellectual faculties appeared to be complete. He enjoyed the use of all his senses, and according to the information gathered at the Chapel near Paris, where he lived, Augé had an ordinary intelligence and walked with the help of a stick until the moment when heart disease forced him to bed. He died two days after his entrance into the hospital, and his hard cadaver [was] examined $28 \mathrm{~h}$ after his death. [The general autopsy showed] thickening of the
\end{abstract}




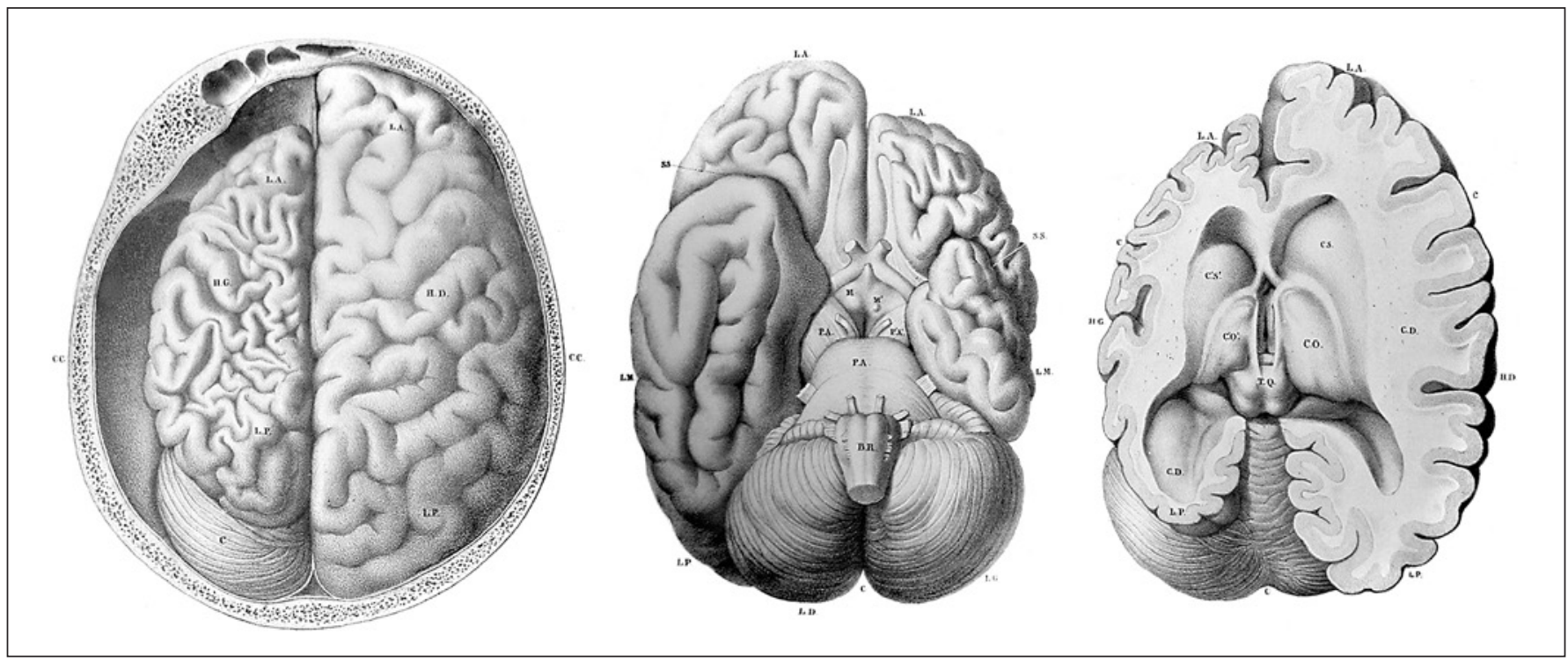

Fig. 3. Images from Plate V, Issue 8, of Cruveilhier's Anatomie Pathologique du Corps Humain (1829-1853) showing findings consistent with Dyke-Davidoff-Masson syndrome, including cerebral hemiatrophy, ipsilateral calvarial thickening (due to widening of diploe, the spongy cancellous bone separating the inner and outer layers of the cortical bone of the skull), hyper-pneumatization of the ipsilateral frontal sinuses, and crossed cerebellar atrophy. The original lithographs were created by French painter and anatomical illustrator Antoine Chazal (1793-1854) but were not characterized by strict realism, given that the opened skull is represented with a smooth edge as if cut by a saw, when instead the

walls of the left ventricle of the heart, which had a volume three to four times greater than in the normal state....

There was a great deal of difficulty in opening the skull by breaking it round with a hammer.... [The] gyri of the left hemisphere [were] thinned, flattened, more conspicuous, and whiter than in the normal state, leaving between them broad and deep sulci... A manifest fluctuation at the slightest touch indicated the presence of a large amount of liquid in the corresponding ventricle. In fact, since the brain had been removed from its bone box [cranial cavity], a very large quantity of clear cerebrospinal fluid had subsided in a few moments, and the left hemisphere, sinking under the eyes, had [been] reduced to below a third of the volume maintained by the [right] hemisphere. ... The difference between the two hemispheres of the brain on the one hand and the thickness of the walls of the skull on the other is also perfectly indicated ... (in Fig. 3). Strange thing! The right lobe ... of the cerebellum is less voluminous than the left lobe of the brain, which would seem to indicate a crossed effect between the brain [cerebral hemisphere] and the cerebellum; but the diminution of the right lobe of the latter cannot be balanced with [i.e., is not the same magnitude as] the atrophy of the left hemisphere of the brain. ... The corpus striatum, thalamus, and cerebral peduncle were also atrophied on the same side... [2, Translation by DJ Lanska]. skull was "opened by breaking it round with a hammer." These images were created approximately a century before the report of Dyke, Davidoff, and Masson in 1933. For this figure, the images were converted to black and white, separated from the background, adjusted for contrast, repaired where damaged, and organized into a linear sequence using Apple Aperture v3.6 and Adobe Photoshop CS6 v13.0. Courtesy of the John Martin Rare Book Room, University of Iowa Libraries. The original plate is in the public domain and is available at the Iowa Digital Library http:// digital.lib.uiowa.edu/cdm/compoundobject/collection/jmrbr/ id/2422/show/2193.

\section{Cruveilhier's Reflections}

Cruveilhier was particularly struck by the apparent dissociation between the marked cerebral hemiatrophy in this case and the patient's relatively good functional level, as demonstrated by his apparently normal cognition and his ability to walk, albeit with the aid of a stick. How was this possible, Cruveilhier wondered, when in other cases, relatively small lesions produce much more dramatic cognitive and motor impairments? To reconcile these observations, Cruveilhier first postulated that some pathologies (e.g., extrinsic compression) produce a complete disruption of nervous pathways, precluding nervous transmission and thereby causing profound impairments, whereas with atrophic processes, the nervous pathways are relatively preserved, even if "imperfect," allowing relatively normal function.

There was atrophy as complete as possible of the whole left hemisphere, and yet the hemiplegia was incomplete on the right. The patient could walk with a stick. How can we reconcile this with other facts that can be seen every day, which show us small effusions or small tumors in the brain producing a com- 
plete hemiplegia of feeling and movement [sic, hemianesthesia and hemiplegia]? I think I discovered its cause in the difference which exists, on the one hand, between atrophy of the brain by compression and atrophy without compression, and, on the other hand, between this atrophy, whatever its cause, and the solution of continuity or the interruption of the cerebral fibers. In atrophy, the instrument of our sensations and volitions is doubtless imperfect; but it exists with all its constituent parts, which fulfill, as well as possible, the functions, which have been devolved to them. In the solution of continuity, on the contrary, a certain number of medullary bundles of the brain are incapable of transmitting the orders of the will and the impressions from without: there is a hiatus, an interval which nothing can cross [2].

Cruveilhier recognized that other cases with "atrophy" of the brain showed much greater impairment than did Augé, and in particular an earlier congenital case of his with apparent atrophy was profoundly mentally retarded. In the case of Augé, Cruveilhier noted that,

An entire hemisphere was atrophied, reduced to a quarter of its volume, and yet the patient enjoyed the integrity of his intellectual faculties: he was endowed with an ordinary intelligence. This case is, therefore, in opposition to that represented in the book [Anatomie Pathologique du Corps Humain, Livraison V, Plate IV], which has offered us an example of the most absolute idiocy produced by the atrophy of about two-thirds of the left hemisphere of the brain although the right hemisphere was also abnormal [2].

To account for these differences, Cruveilhier further postulated a pathogenetic dichotomy of cerebral atrophies, with one group resulting from tissue destruction, and the other with relatively preserved integrity of the cerebral structures, resulting, for example, from a decrease in intracellular and interstitial fluid, or from impaired metabolism.

How can we explain this difference? Let us first distinguish between atrophies: some are the destruction, a loss of substance, or a real transformation of the tissue of the organ, [while] others are the result of a pure and simple diminution of the volume of the organ, either by the subtraction of a liquid which penetrates the meshes of the cellular tissue, or by the approximation of the fibers of the organ without increase of density, or, finally, by the want of nutrition of the fibers themselves. Now the atrophy of the brain, represented by Plate IV [Livraison V - Cruveilhier's earlier case with profound mental retardation], must be classified in the category of atrophies with destruction and transformation, while that of the brain represented here is evidently in the class of atrophies [characterized] by lack of nutrition. In a word, in the latter case no cerebral fiber was missing, strictly speaking: they were all shrunken, atrophied, in the same way as the fibers of the lungs are shrunken and strongly pressed against one another ... in the case of serous or purulent effusion [2].

Cruveilhier's Case of Dyke-Davidoff-

Masson Syndrome
Cruveilhier dismissed a unilateral hydrocephalus as the cause of the atrophy, and instead considered the ventricular enlargement to be an ex vacuo phenomenon resulting from loss of brain tissue. He further speculated that the ipsilateral calvarial thickening was a consequence of the cerebral hemiatrophy.

The cerebrospinal fluid, which distended the lateral ventricle of the corresponding hemisphere, and which filled the meshes of the subarachnoidal cellular tissue [i.e., the subarachnoid space], seems to me the effect, and not at all the cause, of the atrophy... Perhaps the considerable thickening of the bones of the skull on the side of the atrophy is still an effect of this atrophy? [2].

Cruveilhier correctly recognized that the pathologic process occurred early in life, given the osseous changes in the skull, but also noted that those alone could not distinguish intrauterine and postpartum causes.

The question of whether the disease is congenital, or whether it occurred after birth, cannot be resolved by the condition of the bones of the skull. There has been no stopping of development, as the brain is as developed as possible. There was, therefore, a disease, [caused by] lack of nutrition of the brain, and it is irrelevant to the substance of the question whether this lack of nutrition occurred during intrauterine or extrauterine life [2].

Although Cruveilhier had noted with some surprise the crossed cerebellar atrophy in his report of the autopsy findings in this case and had correctly surmised a "crossed effect" between the cerebral hemisphere and the cerebellum, he chose not to elaborate on that in his reflections. $\mathrm{He}$ also recognized the decreased size of the ipsilateral basal ganglia, thalamus, and cerebral peduncle, which are evident in the plate (Fig. 3, center and right), but he did not further elaborate on the significance of this either.

\section{Later Descriptions of Dyke-Davidoff-Masson \\ Syndrome}

In 1933, radiologist Cornelius G. Dyke (1900-1943), neurosurgeon Leo M. Davidoff (1898-1975) and neurosurgeon Clement M. Masson, from the X-ray and surgical departments of the Neurological Institute of New York, described the plain skull radiographic and pneumoencephalographic changes in a series of 9 patients with infantile hemiplegia, noting particularly a variety of osseous and cerebral changes ipsilateral to the cerebral lesion: (1) dilation of the lateral ventricle and variable sulcal enlargement or abnormal collections of air in the subarachnoid space reflecting cerebral hemiatrophy, (2) thickening of the skull, and (3) hyper-pneumatization of the frontal and ethmoid sinuses and of the mastoid air cells - findings 
now referred to as the Dyke-Davidoff-Masson syndrome [4]. Clinically, the cases were characterized by hemiparesis, seizures, and variable degrees of mental retardation (though one was mentally normal) [4]. Dyke and colleagues were apparently unaware of Cruveilhier's case of this syndrome as it was published in a limited edition in French a century earlier.

In 1939, neurologist and neuropathologist Bernard J. Alpers (1900-1981) and Richard B. Dear, from the Laboratory of Neurosurgery at the University of Pennsylvania, reviewed prior reports of cerebral hemiatrophy and divided them into primary and secondary forms [5]. Primary cases largely resulted from hereditary and congenital factors, while secondary cases resulted from a variety of postnatal conditions, most commonly encephalomyelitis and less commonly a cerebrovascular event, inflammatory process, or cranial trauma in early childhood. As part of their report, Alpers and Dear reviewed some of the earlier cases of cerebral hemiatrophy, including a second-hand report of Cruveilhier's case, which they found summarized in a thesis by French neurologist Jules Cotard (1840-1889) [6]. Cotard had been an interne at the Salpêtrière, when he wrote his thesis on cerebral atrophy [6]; the thesis relied heavily on the work of Cruveilhier and included a summary of Cruveilhier's methods of study, his approach to interpretation of pathologic findings, and several of his cases (only one of which can be regarded as a case of cerebral hemiatrophy).

\section{Discussion}

Cruveilhier's Anatomie Pathologique du Corps Humain (1829-1842) "contains some of the finest illustrations of gross pathology ever made" ([7], p. 459), remains "one of the greatest works of its kind" ([8], p. 360), and, as Jean-Martin Charcot (1825-1893) cautioned, it is "an admirable work, which ought to be more frequently consulted by all who desire to avoid the disappointment of making second-hand 'discoveries' in morbid anatomy" ([9], p. 158). The truth of Charcot's sage advice is exemplified by Cruveilhier's long-forgotten case of cerebral hemiatrophy [Livraison VIII, Plate V], an early well-documented case of what is now called Dyke-Davidoff-Masson syndrome [2]. This case was originally published as part of the eighth of twenty fascicles that were issued from 1829 to 1835 and ultimately comprised the first volume of Cruveilhier's atlas; because Augé died in 1830 and because Cruveilhier's output seems to have been fairly steady over even the longer interval of 40 fascicles issued from 1829 to 1842 for both volumes, fascicle VIII was probably initially published in late 1830 or 1831 . As was well documented in the case description and as was beautifully illustrated in the remarkably detailed accompanying lithographic plate (Fig. 3), this case manifested all of the key osseous and cerebral features of the Dyke-Davidoff-Masson syndrome as subsequently elaborated a century later by Dyke and colleagues, including (1) cerebral hemiatrophy with ex vacuo dilation of the lateral ventricle, (2) ipsilateral thickening of the diploe of the skull, and (3) ipsilateral hyper-pneumatization of the frontal sinuses [4]. Dyke and colleagues did not reference Cruveilhier's case and were apparently unaware of it, but in 1939, Alpers and Dear came across a summary of it in Cotard's 1868 thesis (but even they did not seek out the original publication) [4-6].

Cruveilhier's case is somewhat atypical clinically because Augé was cognitively normal and had no reported seizures, whereas the "the usual clinical picture is that of hemiplegia, convulsions, and mental deficiency" ([5], p. 667). In the literature review of Alpers and Dear reported in 1939, "All the cases had a hemiplegia... Most of these cases have convulsions, and almost all of them are mentally deficient, sometimes profoundly so" ([5], p. 667). In the series by Dyke and colleagues, only 1 of 9 (11\%) was considered cognitively normal, and all of the cases had seizures [4]. However, in a more recent series of 10 cases studied by computed tomography, 6 (60\%) were considered cognitively normal and $9(90 \%)$ had a history of seizures, though the only case without a history of seizures was still only 1 year old [10]. In some cases of Dyke-Davidoff-Masson syndrome, the onset of seizures can be delayed into adulthood $[10,11]$, and some cases who were only diagnosed in adulthood did not have a reported history of seizures, including one case diagnosed in her fifties [12].

Cruveilhier's "reflections" on the unexpected clinical findings (i.e., preserved cognition and partially spared contralateral motor function despite such profound cerebral hemiatrophy) suggested a useful framework for categorizing types of cerebral pathologies: by the processes involved and the degree to which they caused tissue destruction or disrupted neural pathways. This straightforward approach had considerable explanatory power and provided a reasonable resolution to otherwise discordant observations. For example, Cruveilhier's approach readily explained how a small strategically placed lesion could cause profound motor impairment, while a much larger lesion of the same hemisphere did not. 
Cruveilhier also made one surprising observation that he chose not to elaborate on in his reflections. In noting the crossed cerebral-cerebellar atrophy in his case, Cruveilhier exclaimed "Strange thing!" and then correctly inferred a "crossed effect" between the cerebral hemisphere and the cerebellum. Crossed cerebellar atrophy typically occurs with long-standing, extensive, unilateral cerebral lesions with onset either in utero or during early childhood [11, 13-15]. Alpers and Dear identified reports of crossed cerebral-cerebellar atrophy among 14 of 24 cases (58\%) of cerebral hemiatrophy, but in the remaining cases, no mention of the cerebellum was made, so it is likely that the true proportion with crossed cerebral-cerebellar atrophy is much higher [5]. As in Cruveilhier's case, several recent pathological and imaging studies of cerebral hemiatrophy have also noted atrophy of both the ipsilateral cerebral peduncle and the contralateral cerebellar hemisphere (with severe loss of cerebellar granule cells and parallel fibers, but with minimal loss of Purkinje cells), supporting a pathophysiologic mechanism involving transneuronal degeneration along the corticopontocerebellar pathways: under this scenario, cerebral hemiatrophy causes a decrease in output through the corticopontine pathway, which produces secondary antegrade transneuronal atrophy of the ipsilateral pontine nuclei (i.e., transsynaptic degeneration or "dying forward," caused by loss of neuronal inputs), which in turn causes a decrease in output along the pontocerebellar pathway and a resulting tertiary antegrade transneuronal degeneration of the contralateral cerebellar granule cell layer $[11,16$, 17]. In some cases, retrograde transneuronal degeneration (i.e., "dying backward" caused by lack of trophic support from the target neurons) within the cerebellorubrothalamic tract following unilateral thalamic and frontal cerebral lesions may also contribute to crossed cerebral-cerebellar atrophy [18]. In other cases, crossed cerebellar atrophy may result from postictal cerebellar lobular sclerosis (with severe loss of Purkinje cells and sparing of the middle cerebellar peduncle) $[13,19]$, although that mechanism cannot be readily implicated in Cruveilhier's case with evident involvement of the ipsilateral cerebral peduncle and in the absence of a history of seizures.

\section{Conclusion}

Cruveilhier's pathological case from 1830 clearly anticipated the cases reported by Dyke and colleagues based on pneumoencephalography and modern case reports, now typically recognized with computed tomography or magnetic resonance imaging. Cruveilhier's case also emphasizes the dubious concept of a "first report" and the importance of considering reports outside of one's current time period and reports written in other than one's native language.

\section{Statement of Ethics}

This study does not require ethical approval.

\section{Conflict of Interest Statement}

The author has no conflicts of interest to declare.

\section{Funding Sources}

The author did not receive any funding.

\section{Author Contributions}

D.L. conceptualized the study and drafted the manuscript for intellectual content.

\section{References}

1 Spillane JD. The doctrine of the nerves: chapters in the history of neurology. Oxford, New York, and Toronto: Oxford University Press; 1981.

2 Cruveilhier J. Anatomie pathologique du corps humain, descriptions, avec figures lithographiées et coloriées, des diverses altérations morbides dont le corps humain est susceptible [Pathological anatomy of the human body: descriptions, with lithographed and colored figures, of various morbid alterations to which the human body is susceptible]. Vol. 1. Paris: J.B. Baillière; 1829-1842.
3 Flamm ES. The neurology of Jean Cruveilhier. Med Hist. 1973;17(4):343-55.

4 Dyke CG, Davidoff LM, Masson CB. Cerebral hemiatrophy with homolateral hypertrophy of the skull and sinuses. Surg Gynecol Obstet. 1933; $57: 588-600$.

5 Alpers BJ, Dear RB. Hemiatrophy of the brain. J Nerv Ment Dis. 1939;89:653-71.

6 Cotard J. Étude sur L'Atrophie Partielle du Cerveau. [Thèse de Paris]. Paris: LeFrançois; 1868.
Cruveilhier's Case of Dyke-Davidoff-

Masson Syndrome
Eur Neurol 2021;84:300-306

DOI: $10.1159 / 000515808$ 
7 Eimas R, editor. Heirs of hippocrates: the development of medicine in a catalogue of historic books in the Hardin library for the health sciences, the University of Iowa. Iowa City, IA: University of Iowa Press; 1990, p. 459.

8 Norman JM, editor. Morton's medical bibliography: an annotated check-list of texts illustrating the history of medicine (Garrison and Morton). 5th ed. Brookfield, VT: Scholar Press, Gower Publishing Company; 1991. p. 360.

9 Charcot JM. Lectures on the diseases of the nervous system delivered at la Salpêtrière. Translated by George Sigerson. London: The New Sydenham Society; 1877. p. 158.

10 Zilkha A. CT of cerebral hemiatrophy. AJR Am J Roentgenol. 1980;135(2):259-62.
11 Winkler DT, Probst A, Wegmann W, Tolnay M. Dyke-Davidoff-Masson syndrome with crossed cerebellar atrophy: an old disease in a new millenium. Neuropathol Appl Neurobiol. 2001;27(5):403-5.

12 Biçici V, Ekiz T, Bingöl I, Hatipoğlu C. DykeDavidoff-Masson syndrome in adulthood: a 50-year diagnostic delay. Neurology. 2014; 83(12):1121.

13 Strefling AM, Urich H. Crossed cerebellar atrophy: and old problem revisited. Acta Neuropathol. 1982;57:197-202.

14 Tien RD, Ashdown BC. Crossed cerebellar diaschisis and crossed cerebellar atrophy: correlation of MR findings, clinical symptoms, and supratentorial diseases in 26 patients. AJR Am J Roentgenol. 1992;158(5):1155-9.
15 Chakravarty A. MR evaluation of crossed and uncrossed cerebral-cerebellar diaschisis. Acta Neurol Scand. 2003;108(1):60-5.

16 Cowan WM. Anterograde and retrograde transneuronal degeneration in the central and peripheral nervous system. New York: Springer; 1970. p. 217-51.

17 Gupta R, Joshi S, Mittal A, Luthra I, Mittal P, Verma V. Magnetic resonance imaging depiction of acquired Dyke-Davidoff-Masson syndrome with crossed cerebro-cerebellar diaschisis: report of two cases. J Pediatr Neurosci. 2015;10(3):294-6.

18 Chung HD. Retrograde crossed cerebellar atrophy. Brain. 1985;108(Pt 4):881-95.

19 Tan N, Urich H. Postictal cerebral hemiatrophy: with a contribution to the problem of crossed cerebellar atrophy. Acta Neuropathol. 1984;62(4):332-9. 